\title{
Parallel Approaches to the Integration of the Differential Equations for Reactive Scattering
}

\author{
Valentina Piermarini, Leonardo Pacifici, Stefano Crocchianti and Antonio \\ Laganà
}

Dipartimento di Chimica, Università di Perugia, Via Elce di Sotto, 8, 06123 Perugia, Italy

\begin{abstract}
Parallel restructuring of computational codes devoted to the calculation of reactive cross sections for atom-diatom reactions plays an important role in exploiting the potentialities of concurrent platforms. Our reactive scattering codes have been parallelized on different platforms using MPI and performances have been evaluated to figure out the most efficient organization models. The same codes have been used for testing new parallel environments and related coordination languages.
\end{abstract}

\section{Introduction}

Properties of elementary chemical reactions [1] and related virtual reality constructions [2] can be determined accurately by using rigorous quantum methods to solve the Schrödinger equation. After separating the electronic motion, the motion of the nuclei can be described by a set of time dependent Schrödinger equations:

$$
\hat{H}_{n}(\{\mathbf{W}\}) \Psi_{n}(\{\mathbf{W}\}, t)=i \hbar \frac{\partial}{\partial t} \Psi_{n}(\{\mathbf{W}\}, t)
$$

where $t$ is time, $\{\mathbf{W}\}$ is the set of nuclear coordinates, $\hat{H}_{n}$ is the Hamilton operator and $\Psi_{n}(\{\mathbf{W}\}, t)$ is the time dependent nuclear wavefunction (from now on the index $n$ will be dropped by considering only the case of the ground electronic state). The solution of this differential equation delivers the information necessary to rationalize the dynamics of the reaction considered and to evaluate its measurable properties. Such a solution can be obtained either by choosing time as a continuity variable and integrating directly the time dependent formulation of the Schrödinger equation or by factorizing the time dependence in the system wavefunction and integrating the related stationary formulation. As a case study we consider here the atom diatom systems for which is:

$$
\hat{H}\left(\mathbf{R}_{\tau}, \mathbf{r}_{\tau}\right) \Psi\left(\mathbf{R}_{\tau}, \mathbf{r}_{\tau}, t\right)=\left[-\frac{\hbar^{2}}{2 \mu}\left(\nabla_{\mathbf{R}_{\tau}}^{2}+\nabla_{\mathbf{r}_{\tau}}^{2}\right)+V\left(R_{\tau}, r_{\tau}, \Theta_{\tau}\right)\right] \Psi\left(\mathbf{R}_{\tau}, \mathbf{r}_{\tau}, t\right)
$$

where $\mathbf{R}_{\tau}$ and $\mathbf{r}_{\tau}$ are the mass scaled atom-diatom Jacobi coordinates of arrangement $\tau, \Psi\left(\mathbf{R}_{\tau}, \mathbf{r}_{\tau}, t\right)$ is the nuclear wavefunction and $V\left(R_{\tau}, r_{\tau}, \Theta_{\tau}\right)$ is the potential expressed in terms of $R_{\tau}$ and $r_{\tau}$ (the moduli of $\mathbf{R}_{\tau}$ and $\mathbf{r}_{\tau}$ ) and of the 
angle $\Theta$ formed between them. In practice, by exploiting the properties of the rotations of the rigid bodies the dimensionality of the problem can be further reduced by formulating also the hamiltonian and the wavefunctions in terms of $R_{\tau}, r_{\tau}$ and $\Theta$ (or the corresponding hyperspherical coordinates, like the APH3D ones $\rho, \theta$ and $\chi[3])$ and the three Euler angles $\alpha, \beta$ and $\gamma$.

\section{Time dependent versus time independent approaches}

Time dependent approaches to the solution of the Schrödinger equation are conceptually quite simple since they set the system wavefunction in its initial configuration and then let it evolve in time under the effect of the Hamiltonian operator. The scheme of the time dependent code (TIDEP) is shown below. The outcome of the calculation depends on the initial conditions chosen. In the scheme the $\mathrm{C}$ coefficients represent the matrix elements which are manipulated

Read input data

\section{SECTION I}

Set inizialization and initial conditions

Calculate auxiliary variables

LOOP on $\mathrm{t}$

SECTION II

Perform the propagation step

Store the $\mathrm{C}$ coefficients

END LOOP on $t$

SECTION III

Calculate final quantities from $\mathrm{C}$ coefficients and print the output

Fig. 1. Scheme of the TIDEP program

in SECTION III in order to obtain dynamical information. Time independent approaches to the solution of the Schrödinger equation have a more complex structure. They are typical irregular problems. The related hyperspherical coordinate computational procedure, APH3D, is articulated into several computer programs. The first of these programs is ABM. ABM calculates the eigenvalues and the eigenfunctions $\Phi$ of the hyperangular (in $\theta$ and $\chi$ ) part of the hamiltonian for all the sectors in which the hyperradius $\rho$ has been partitioned. The code then assembles the coupling matrix for the propagation on $\rho$ to be performed at a fixed value of the total energy by the second program LOGDER. These two programs are the most time and memory consuming components of the computational procedure. The schemes of the ABM and LOGDER programs are given in Fig. 2 and Fig. 3, respectively. 
Input general data

\section{SECTION I}

Calculate quantities of common use

LOOP on sectors

\section{SECTION II}

Calculate the value of $\rho$ at the sector midpoint LOOP on $\Lambda$

Calculate eigenvalues and surface functions

Store on disk eigenvalues

IF(not first $\rho$ )

THEN

Calculate overlaps with previous sector functions

Store on disk the overlap matrix

\section{END IF}

END the $\Lambda$ loop

LOOP on $\Lambda$

Calculate the coupling matrix

END the $\Lambda$ loop

Store on disk the coupling matrix

END the sector loop

Fig. 2. Scheme of the ABM program.

Input general data

\section{SECTION I}

Read from disk data stored by ABM

Calculate quantities of common use

LOOP on $N_{E}$ energies

\section{SECTION II}

Embed the energy dependence into the coupling matrix

LOOP over sectors

Single step propagate the fixed $J$ and $p$ logarithmic derivative matrix

END the sector loop

Calculate and store the final logarithmic derivative matrix elements on disk END the energy loop

Fig. 3. Scheme of the LOGDER program. 
As is apparent from the schemes of Fig. 1 and Fig. 3 a critical point of both TIDEP and LOGDER calculations is the fact that the natural computational grain is the fixed $J(J$ is the total angular momentum quantum number whose projections on the quantization axis are the $J+1$ values of $\Lambda$ ) and $p$ (parity) integration of the scattering equations.

\section{Data structure and Data streams}

The flux diagram of ABM is given in Fig. 4. In the figure the nodes represent

\begin{tabular}{|c|}
\hline M1 \\
\hline M2 \\
\hline M3 \\
\hline M4 \\
\hline
\end{tabular}
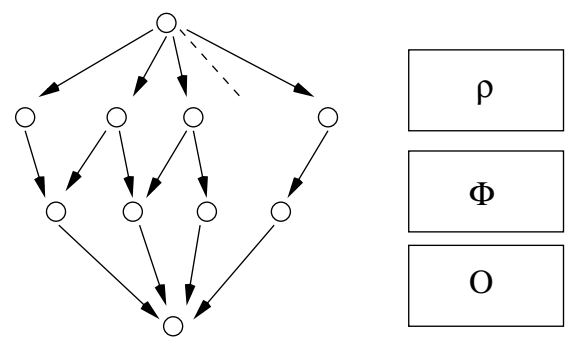

Fig. 4. ABM application graph

the computational moduli while the arches represent fluxes of data. The name of the moduli are given in the left hand side boxes while the names of the matrices containing the key information are given in the right hand side boxes. In particular, the first relevant data are the surface functions $\Phi$, in which the fixed $J$ and $p$ partial wave has been expanded and that are calculated at the midpoint value of $\rho$ for each sector. The other relevant data are the overlap matrix $\mathbf{O}$ and the coupling matrix $\mathbf{D}$ that are stored on disk for further use by LOGDER. The flux diagram indicates that the M1 module reads the input data and performs preliminary calculations of general use including the partitioning of $\rho$ into sectors. The M2 module calculates then the surface functions $\Phi$ and sends them to module M3 to calculate the overlap matrix between the surface functions of adjacent sectors at the common border. The calculation of these overlap integrals introduces an order dependence that can be solved in different ways. Additional calculations are performed by the sequential module M4 in order to calculate the coupling matrix $\mathbf{D}$ out of the vector eigenvalues and overlap matrices. The D matrix is then stored on disk for a subsequent use by the LOGDER program. The flux diagram of the second program, LOGDER, which carries out the propagation of the solutions of the scattering equations at a fixed value of energy E, is given in Fig. 5. For this program the most important data are the coupling matrix $\mathbf{D}$ and the solution matrix $\mathbf{R}$, either at the final integration point (Rf) or at its asymptotic limit (Ra). The flux diagram indicates that the M1 module after reading the input data (and in particular the coupling matrix $\mathbf{D}$ ) and carrying out the preliminary calculations of general variables, passes the $\mathbf{D}$ 


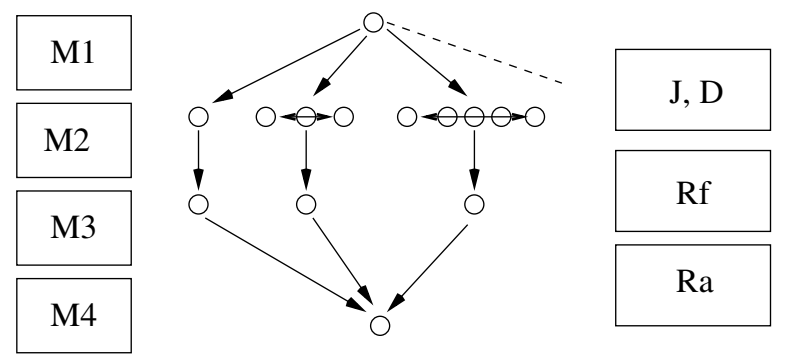

Fig. 5. LOGDER application graph

matrix to the next module together with the value of the total angular momentum quantum number $J$, on which depends the number of projections $\Lambda$ to be considered. This is the key quantity of the calculation since it determines in the module M2 the number of blocks of the $\mathbf{R}$ matrix which during the propagation along the reaction coordinate need to communicate (they are, in fact, tightly coupled and do not allow a coarse grain parallel model to scale, due to the increase with the $J$ value of the memory required). At the end of the integration, the $\mathbf{R f}$ matrices are passed to the nodes M3 to be transformed into Ra matrices which are then passed to module M4 to be assembled and stored for further use. Despite the several conceptual differences, the same graph applies to TIDEP as also apparent from the schemes of Figs. 1 and 3 .

\section{Message passing parallelization}

The parallel organization of the computational moduli as well as the management of the fluxes of data described above, have been implemented using MPI. The model used for the ABM program is a dynamic task-farm in which the calculation of the surface functions $\Phi$ of each sector is assigned to the first available node by a dynamic allocation controlled by the master. The surface functions are then used for calculating the $\mathbf{O}$ matrix of the overlap integrals between surface functions of adjacent sectors at the common border and for the construction of the coupling matrix D. In this case shared memory would allow an access to data of different sectors in the sequence dictated by the order in which overlaps between functions of adjacent sectors need to be calculated. Using the message passing paradigm of MPI this can be obtained by the (repeated) direct calculation of the surface functions when needed, either by solving again the Schrödinger equation for the previous sector, or in a simpler way, by combining the primitive functions using the already calculated eigenvectors. This choice, together with a dynamic distribution of the work, greatly reduces the communication time and minimizes the work unbalance. Accordingly, the master reads the input data and distributes them to the slaves as soon as they are available and ready to start a new job. The MPI structure of the ABM master is shown in Fig. 6.

On their side, the slaves receive at first the input data and afterwards the $\rho$ value 
Read input data

\section{MASTER PROCESS}

Send input data to the slaves

LOOP on sector index

Calculate the value of $\rho$ at the sector midpoint

Call MPI_SEND $(\rho)$

END LOOP on sector index

Fig. 6. Pseudo MPI code for the master process in ABM

associated with the sector for which they have to calculate the surface functions $\Phi$. The slaves (see in Fig. 7 the related MPI structure), after calculating the surface functions, write on disk the eigenvectors, which are the coefficients of the surface functions in the given basis set. When the sector is not the first one,

Receive input data

\section{SLAVE PROCESS}

10 Call MPI_RECV $(\rho)$

LOOP on $\Lambda$

Construct the primitive basis set at the given value of $\rho$

Solve the angular Schrödinger equation by expanding in the primitives

Store on disk eigenvalues and eigenvectors

IF(not first sector) then

Construct the basis set at the previous value of $\rho$

Read from disk related eigenvectors

Compute overlap integrals at the common edge of the two sectors

END IF

END LOOP on $\Lambda$

Calculate the coupling matrix

Store on disk the coupling matrix for use from LOGDER

GOTO 10

Fig. 7. Pseudo MPI code for the slave process in ABM

the process has to be repeated (or corresponding eigenvectors have to be read from disk). This allows the calculation of surface functions of the previous sector, and the evaluation of the overlap integrals between them. Then, the coupling matrix is calculated and stored on disk, together with the overlap matrix, for use by the LOGDER program. This parallel model has been implemented on the CRAY T3E of EPCC (Edinburgh, UK) using up to 128 processors. The individual processor computing time never exceed more than $10 \%$ the average one and the speedup was never smaller than $70 \%$ the ideal value [4]. 
A similar task farm model was adopted for LOGDER and TIDEP. However a similar excellent performance was obtained only for the lowest $J=0$ runs, due to the difficulty of handling shared memory with MPI. The only possibility of being able to deal with this class of irregularity in a portable way (the CRAY machine has its own shared memory environment) is to turn into abstract level coordination languages.

\section{The coordination language parallelization}

A very promising coordination language being developed by prof. Vanneschi and collaborators at the Department of Informatics of Pisa is ASSIST [5]. It sprouts out of the skeleton based language SkIE (Skeleton-based Integrated Environment) [6]. SkIE defines some moduli (called skeletons) structured as parallel model prototypes that can be used as building blocks of a parallel application. ASSIST introduces a more general and flexible module, called parmod, whose structure can be defined by the user by making reference to a set of virtual processors (VP) as well as a tool allowing the use of external objects (variables, routines, etc.) and emulating the shared memory. The use of these concepts has allowed us to develop several constructs to implement ABM and it has made possible also a portable parallelization of LOGDER and TIDEP for an arbitrary value of $J$. As for ABM, see Fig. 8, the first model is straightforwardly derived by

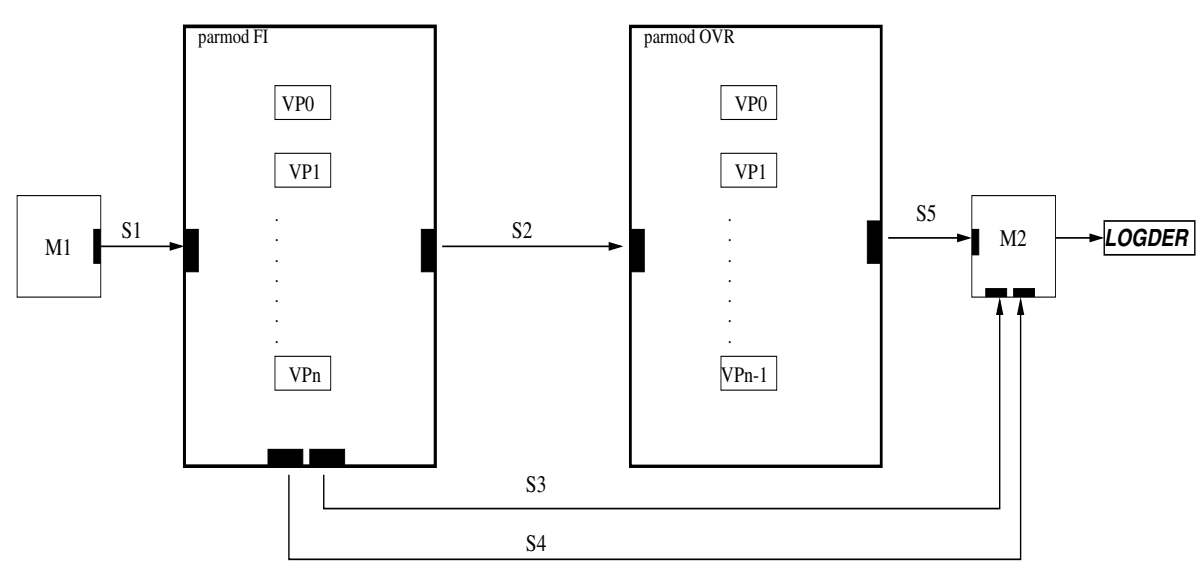

Fig. 8. ASSIST scheme of the first model for ABM

a message passing implementation of the sequential version and is characterized by four moduli: a sequential (M1) and three parallel (FI, OV and M2) ones. M1 executes some preliminary calculations and sends the value of the reaction coordinate of each sector to parmod FI, using the output stream S1 (indicated in the figure as an arrow connecting M1 to F1). The VPs of parmod F1 are arranged 
in a monodimensional array and calculate the surface functions by solving the Schrödinger equation on the hyperangles. Eigenvalues and components of the coupling matrix built out of the surface functions of the same sectors are sent to parmod M2 by the output streams S3 and S4. Surface functions are sent to the parmod OV through the output stream S2 to calculate the overlap matrix O between surface functions of adjacent sectors. The parmod OV, that has the same topology as FI, after receiving the values of the surface functions of the involved sectors at the quadrature points needed for the evaluation of the overlap, builds the matrix $\mathbf{O}$ to be used for mapping the $\mathbf{R}$ matrix from one sector to the next one and sends it to parmod M2 by the output stream S5. The second model is quite similar to the first one (see Fig. 9). In fact it is still constituted by 4 moduli one of which is sequential and three are parallel. The main difference between the two models is that in the latter one the transfer of the values of the surface functions has been replaced by a transfer of the eigenvectors and the parameters of the primitive functions through the output streams S2 and S3, as it has been done by using MPI. This enables the next module, parmod OV, to calculate values of surface functions of the previous sector on the quadrature grid points of the current sector and to evaluate the overlap integrals by minimizing communications. The transfer of the coupling matrix terms and eigenvalues is still performed directly using the parallel module M2 (using the output streams S4 and S5) which collects also the output of parmod OV thanks to the output stream S6. The third model is characterized by the fact that there is only

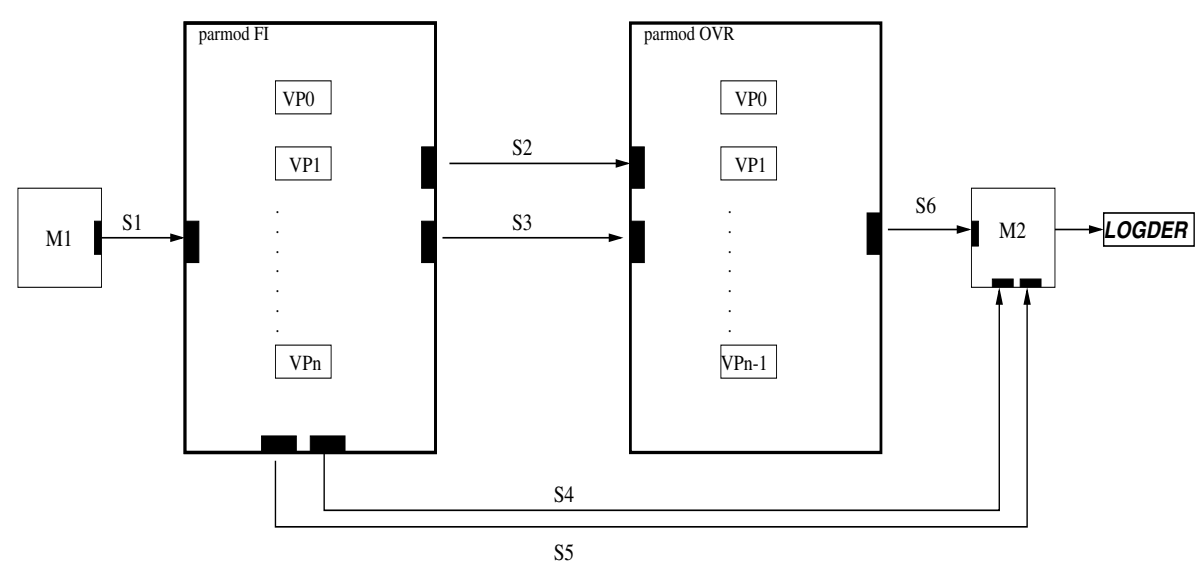

Fig. 9. ASSIST scheme of the second model for ABM

one parmod module placed between the two sequential ones, as can be seen in Fig. 10. While M1 works exactly in the same way as in the first two models, the parmod ABM does not have anymore the structure of a monodimensional array. In fact, in this case, the VPs of the parmod are organized in a $2 \mathrm{xN}$ matrix in which the virtual processors of the first row elaborate the data coming from the 


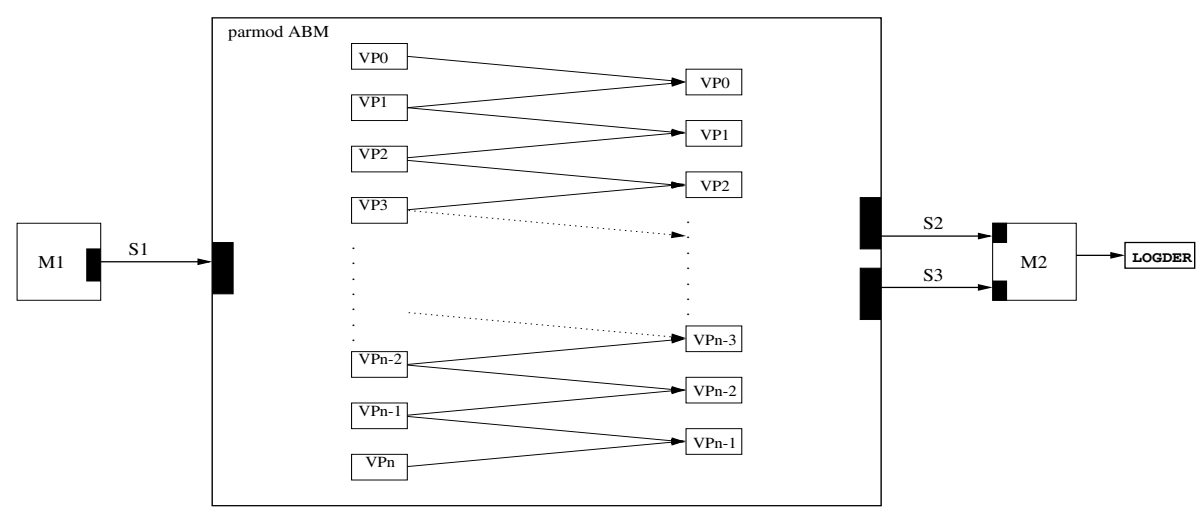

Fig. 10. ASSIST scheme of the third model for ABM

input stream S1 and calculate the surface functions. On the other hand, virtual processors of the second row calculate the overlap integrals on the data coming from the processors of the first row. The transfer of the coupling matrix and of the eigenvalues terms derived from the surface functions of the same sector to module M2 is performed through the output stream S2, while the transfer of the coupling matrix is performed through the output stream S3. The fourth

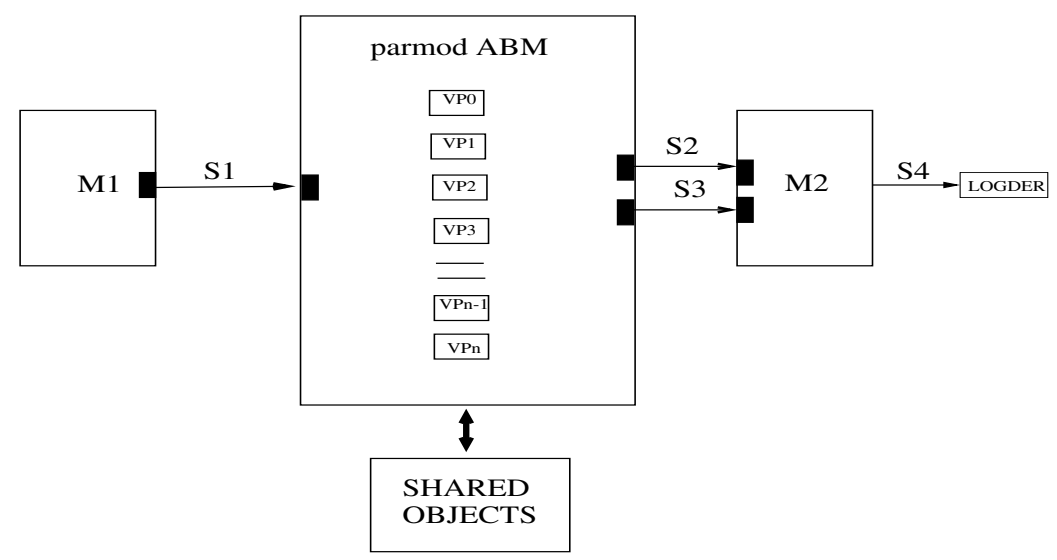

Fig. 11. ASSIST scheme of the fourth model for ABM

model is the simplest one and the closer to the sequential version of the code. It is characterized by a single parmod sandwiched in a PIPE between two sequential modules M1 and M2. The structure of the model is, therefore, identical to that of the third one, yet the array structure of the VPs is monodimensional. This is due to the use of shared objects (see Fig. 11) to handle the sequentiality 
associated with the calculation of overlap integrals between surface functions of adjacent sectors. Data transfers are ensured by streams of output like S1 (from the sequential module M1 to parmod ABM) and S2 and S3 (from parmod ABM to the sequential module M2). Again, S4 sends the coupling matrix $\mathbf{D}$ to the LOGDER program.

A similar extremely simple approach can be adopted for LOGDER and TIDEP. As shown by Fig. 12, the sequential module M1 arranges the $\mathbf{D}$ and $\mathbf{O}$ matrices

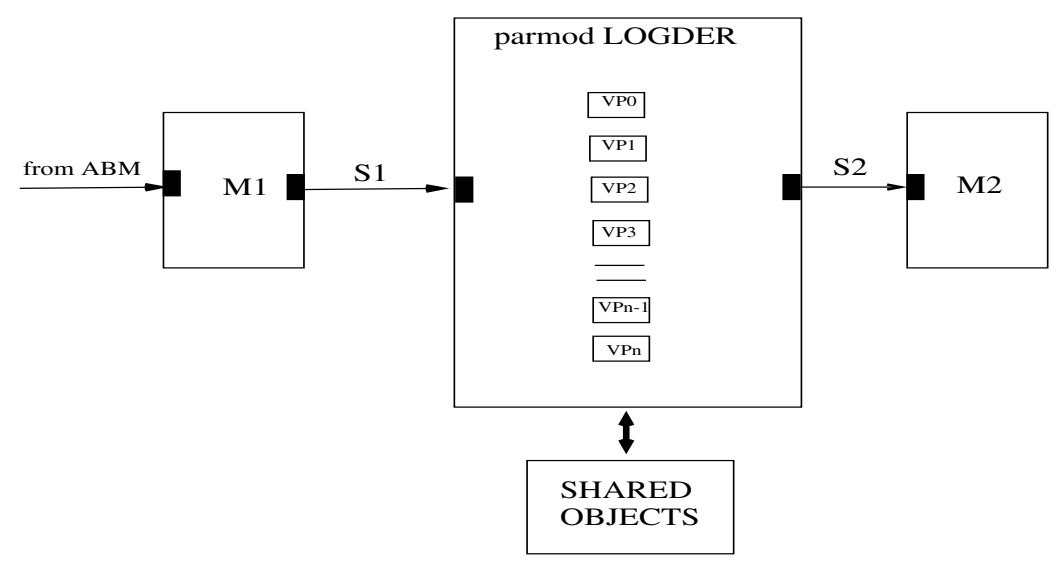

Fig. 12. ASSIST scheme of LOGDER

(read from disk) for use by the pipeline of VPs given in the central parmod LOGDER. Then the resulting $\mathbf{R f}$ matrix final elements are converted into $\mathbf{R a}$ matrix (asymptotic) and passed to subsequent analysis program.

\section{References}

1. Laganà, A., Riganelli, A.: Computational Reaction and Molecular Dynamics: from Simple Systems and Rigorous Methods to Large Systems and Approximate Methods. Lecture Notes in Chemistry, 75 (2000) 1-12

2. Laganà, A., Riganelli, A.: Chimica e Informatica: Dal laboratorio alla realtà virtuale molecolare (Morlacchi, Perugia) (2001) ISBN 88-87716-46-3

3. Parker, G. A., Pack, R. T, Laganà, A., Archer, B. J., Kress, J.D. and Bačic, Z.: Exact quantum results for reactive scattering using hyperspherical (APH) coordinates. Supercomputer Algorithms for reactivity, Dynamics and Kinetics of Small Molecules, A. Laganà Ed. (Kluwer, Dordrecht) (1989) 105-129

4. Laganà, A., Crocchianti, S., Bolloni, A.: Quantum isotopic effects and reaction mechanism: the Li + HF reaction. Phys. Chem. Chem. Phys. 2 (2000) 535

5. Ciullo, P., Danelutto, M., Vaglini, L., Vanneschi, M., Guerri, D., Lettere, M. Progetto ASI-PQE2000, Workpackage 1, Ambiente ASSIST: modello di programmazione e linguaggio di coordinamento ASSIST-CL (versione 1.0) (2001)

6. Vanneschi, M.: Parallel Paradigms for Scientific Computing. Lecture Notes in Chemistry, 751 (2000) 168-181 